\title{
AVALIAÇÃO DE MÉTODOS ALTERNATIVOS DE PREPARO DE AMOSTRAS PARA DETERMINAÇÃO DE CLORETO EM PETRÓLEO
}

\author{
C. M. STRIEDER, S. M. CRUZ, C. K. GIESBRECHT, G. T. DRUZIAN, L. SCHMIDT, \\ E. M. M. FLORES*
}

Universidade Federal de Santa Maria, CEP 97015-900, Santa Maria, RS, Brasil. *ericommf@gmail.com

\begin{abstract}
RESUMO - A presença de cloreto $\left(\mathrm{Cl}^{-}\right)$em petróleo, mesmo em baixas concentrações, pode causar corrosão e prejudicar a qualidade de seus derivados. Nesse sentido, o controle da quantidade de sais é feito em função da quantidade de $\mathrm{Cl}^{-}$. No entanto, o procedimento oficial atual (ASTM D 6470-99) possui algumas desvantagens que o torna inadequado, principalmente para análises em baixas concentrações, indicando assim a necessidade de procedimentos mais apropriados Desta forma, o presente trabalho propõe a utilização de métodos alternativos de preparo de amostras de petróleo: a combustão iniciada por micro-ondas (MIC) e a extração assistida por micro-ondas (MAE), para posterior determinação de $\mathrm{Cl}^{-}$por cromatografia de íons (IC). Foram otimizados alguns parâmetros como a massa de amostra de petróleo (leves e pesados), a ser inserida em cada um dos sistemas. Quando utilizadas amostras de petróleo leve no procedimento de MIC, foi utilizado um retardante de chama (lã de vidro) juntamente com o petróleo. Para avaliar a exatidão dos procedimentos de MIC e MAE, foi utilizado material de referência certificado (CRM) para $\mathrm{Cl}^{-}$e a concordância com o valor informado foi superior a $95 \%$ para ambos os procedimentos. Os limites de quantificação (LOQs) obtidos para MAE foram superiores aos obtidos por MIC, em função da maior massa empregada. Os resultados obtidos indicam que os métodos propostos são adequados para a determinação de $\mathrm{Cl}^{-}$, mesmo que em baixas concentrações.
\end{abstract}

\section{INTRODUÇÃO}

O petróleo é constituído por uma mistura de hidrocarbonetos, oriunda da decomposição da matéria orgânica pela ação da temperatura e pressão ao longo dos anos, encontrada em reservatórios subterrâneos. Mesmo sendo majoritariamente composto por hidrocarbonetos, também podem ser encontrados no petróleo compostos orgânicos contendo $\mathrm{S}, \mathrm{O}, \mathrm{N}$ e elementos metálicos ( $\mathrm{Fe}, \mathrm{V}, \mathrm{Ni}$ e $\mathrm{Cu}$, principalmente), assim como sais e sedimentos. Os diferentes elementos encontrados no petróleo podem ser oriundos do seu processo de formação, de acordo com a origem do óleo, ou ainda incorporados durante o refino (Speight, 2002).

Para o refino do petróleo, inicialmente são obtidas emulsões do tipo água em óleo, que podem ser formadas no interior dos reservatórios ou durante o processo de transporte e prospecção, em função da presença de emulsificantes naturais e do cisalhamento dos fluidos 
imiscíveis. A água emulsificada no óleo é mais difícil de ser removida do que a água livre, que é separada do óleo simplesmente pela ação da gravidade. Na fase aquosa estão presentes sais, na sua maioria cloretos (principalmente $\mathrm{NaCl}$ e menores quantidades de $\mathrm{MgCl}_{2}$ e $\mathrm{CaCl}_{2}$ ) (Bradley, 1992). Estes sais podem ser incorporados no óleo durante a sua formação, no interior das rochas reservatório, e em menores quantidades podem ser agregados durante o processamento do óleo, especialmente em operações do tipo offshore (Fortuny et al, 2008). O tratamento das emulsões reduz os teores de água e sais presentes no petróleo, sendo que diversos métodos para realização desta operação têm sido propostos na literatura (Pereira, 2012). Devido à importância de reduzir estes teores, o processo de dessalga apresenta-se como uma etapa crítica do processamento, uma vez que visa a redução da concentração a níveis entre 1 e $5 \mathrm{mg} \mathrm{kg}^{-1}$ (Morigaki et al., 2010).

A presença de sais com elevados pontos de ebulição no petróleo é prejudicial, mesmo que em pequenas concentrações, uma vez que estes podem se depositar em tubulações e trocadores de calor, dificultando o processo de refino e afetando diretamente o valor do óleo perante o mercado (Gary, 2001). Em elevadas temperaturas, os íons $\mathrm{Cl}^{-}$podem sofrer hidrólise, formando vapores corrosivos no interior de equipamentos, o que também contribui para o aumento dos custos do processo de tratamento do óleo (Speight, 2002). Quando compostos contendo halogênios e metais são transferidos para os derivados do petróleo, como, por exemplo, os combustíveis, estes podem causar envenenamento de catalisadores e corrosão das partes internas dos motores de combustão. A volatilidade dos halogênios, como o cloreto, associada ao elevado consumo de combustíveis fósseis, pode ocasionar também a emissão de quantidades elevadas destes elementos para o meio ambiente (Antes et al., 2010), sendo que, do ponto de vista ambiental, a presença de halogênios contribui para a liberação da forma oxidada do mercúrio na atmosfera (também conhecido como mercúrio gasoso reativo), em óleos com teores mais elevados de Hg (Speight, 2002).

Atualmente, a determinação de cloreto no petróleo é realizada de acordo com o método oficial da ASTM (D 6470-99), que faz uso de titulação potenciométrica, onde o óleo é dissolvido em xileno e posteriormente extraído com álcool, acetona e água em um equipamento de extração sob aquecimento. Dentre as desvantagens observadas nesse método estão o uso de solventes tóxicos, o elevado tempo consumido (mais de $4 \mathrm{~h}$ ), além dos riscos de contaminação, tornando-o assim inconveniente para a determinação de $\mathrm{Cl}^{-}$em baixas concentrações.

Em função da importância do controle de qualidade do petróleo no que se refere ao teor de sais (verificado em função da concentração de $\mathrm{Cl}^{-}$), da complexidade da matriz (elevado teor de matéria orgânica), da volatilidade do analito, das possibilidades de perdas e da susceptibilidade à contaminação (ambiente, materiais e reagentes), faz-se necessário o desenvolvimento de métodos de preparo de amostras adequados. Neste sentido, observando ainda as desvantagens do procedimento padrão de determinação de cloretos em petróleo e os bons resultados obtidos em estudos prévios com métodos alternativos de preparo de amostras em diversos tipos de matriz (Flores et al., 2007; Pereira et al., 2009; Pereira et al., 2010; Antes et al., 2010; Maciel et al., 2014), o presente trabalho tem por objetivo avaliar os métodos de combustão iniciada por radiação micro-ondas e extração assistida por radiação micro-ondas para o preparo de amostras de petróleos pesados e leves, para posterior quantificação de $\mathrm{Cl}^{-}$por IC, em baixas concentrações. 


\section{MATERIAIS E MÉTODOS}

Os métodos de preparo de amostra avaliados no Laboratório de Química Industrial e Ambiental (LAQIA) do departamento de química da Universidade Federal de Santa Maria, foram feitos em um forno de micro-ondas Multiwave $3000^{\circledR}$ (Anton Paar, Áustria). Para este estudo, foram utilizadas amostras de petróleo leve e pesado (18 - $\left.34^{\circ} \mathrm{API}\right)$. Para MIC, as amostras de petróleo pesado $\left(<22^{\circ} \mathrm{API}\right)$ foram envolvidas em um filme de polietileno de uso comercial, colocadas sobre um papel filtro impregnado com $50 \mu \mathrm{L}$ de solução de $\mathrm{NH}_{4} \mathrm{NO}_{3} 6$ mol L-1 (iniciador de combustão) e então posicionadas em um suporte de quartzo que, posteriormente foi colocado em um frasco de quartzo contendo $6 \mathrm{~mL}$ de solução absorverdora $\left(\mathrm{NH}_{4} \mathrm{OH} 10 \mathrm{mmol} \mathrm{L}^{-1}\right)$. O sistema foi pressurizado com $\mathrm{O}_{2}(20$ bar $)$ e submetido à radiação micro-ondas por 5 min (ignição da amostra e etapa de refluxo, $1400 \mathrm{~W}$ ). No caso das amostras de petróleo leve, o procedimento para combustão foi similar, entretanto foi necessária a utilização de lã de vidro, como retardante de chama, juntamente com o óleo. Cabe destacar que todos os materiais utilizados para envolver as amostras (lã de vidro, papel filtro) foram previamente descontaminados com solução de etanol e água em banho de ultrassom. Para o procedimento de MAE, $10 \mathrm{~g}$ de amostra e $20 \mathrm{~mL}$ de água foram transferidos para frascos de quartzo e a radiação micro-ondas foi aplicada por $15 \mathrm{~min}(1200 \mathrm{~W}$, rampa de 10 min, 3 ciclos). Após o programa de aquecimento, a água foi coletada com o auxílio de uma seringa. Para ambos os procedimentos, a determinação de cloreto foi feita por cromatografia de íons (Metrohm, Suíça) com detector de condutividade.

\section{RESULTADOS E DISCUSSÕES}

Em função do comportamento diferenciado das amostras de petróleo leve e pesado no método de MIC, após a realização da otimização da massa a ser inserida no sistema, foi possível decompor até $500 \mathrm{mg}$ de petróleo pesado, enquanto que para a queima de $300 \mathrm{mg}$ de petróleo leve, foram utilizados $100 \mathrm{mg}$ de lã de vidro, para evitar um aumento excessivo na pressão interna dos frascos e possíveis danos ao sistema. Os resultados obtidos para a determinação de $\mathrm{Cl}^{-}$e os limites de quantificação (LOQ) estão mostrados nas Tabelas 1 e 2, respectivamente.

Tabela 1 - Concentração de $\mathrm{Cl}^{-}$em amostras de petróleo $(\mathrm{n}=4)$.

\begin{tabular}{lcc}
\hline \multirow{2}{*}{ Amostras } & \multicolumn{2}{c}{$\mathbf{C l}^{-}\left(\boldsymbol{\mu g ~ g}^{-1}\right)$} \\
\cline { 2 - 3 } & MIC & MAE \\
\hline Petróleo A & $<5,0$ & $1,10 \pm 0,03$ \\
Petróleo B & $66,9 \pm 1,6$ & $65,2 \pm 2,0$ \\
Petróleo C* & $15,8 \pm 0,7$ & $16,8 \pm 0,5$ \\
Petróleo D* & $<8,0$ & $2,48 \pm 0,21$ \\
NIST 1634c** & $47,3 \pm 2,5$ & $43,0 \pm 1,3$ \\
\hline
\end{tabular}

* Petróleo leve

** Valor informado de $\mathrm{Cl}^{-}=45,0 \mu \mathrm{g} \mathrm{g}^{-1}$ 
Tabela 2 - LOQ para os métodos de MIC e MAE em amostras de petróleo.

\begin{tabular}{ccc}
\hline \multirow{2}{*}{ Petróleo } & \multicolumn{2}{c}{$\mathbf{C l}^{-}\left(\boldsymbol{\mu g ~ \mathbf { g } ^ { - 1 } )}\right.$} \\
\cline { 2 - 3 } & MIC & MAE \\
\hline Leve & 8,0 & 0,025 \\
Pesado & 5,0 & 0,025 \\
\hline
\end{tabular}

Não foi observada diferença significativa (nível de confiança de 95\%) quando os resultados para o CRM (NIST 1634c) obtidos pelos métodos de MIC e MAE foram comparados com o valor informado (teste- $t$ ). As demais análises estatísticas foram feitas para as amostras que, por ambos os métodos, puderam ser quantificadas (petróleos B e C), sendo que não foram observadas diferenças significativas quando comparados os resultados obtidos pelas duas metodologias (MIC e MAE). Para isso, a análise estatística foi feita utilizando o software GraphPad InStat (GraphPad InStat Software Imc, Version 3.0, 1997), teste- $t$ pareado, bicaudal. Os valores de LOQ obtidos por ambos os métodos também foram considerados adequados, como descrito na Tabela 2.

\section{CONCLUSÃO}

A combustão iniciada por micro-ondas e a extração assistida por micro-ondas foram adequadas para o preparo de amostras de petróleo leve e pesado para posterior determinação de $\mathrm{Cl}^{-}$por cromatografia de íons, em baixas concentrações. Foi possível observar que quando submetidas à combustão (MIC), as amostras de petróleo leve apresentam comportamento diferenciado das demais, fato este que limita a massa de amostra que pode ser introduzida no sistema e influencia diretamente o LOQ. Quando são utilizadas maiores massas de amostra, a quantidade de gases gerados na decomposição também é acrescida e, no caso de petróleo leve, esse fato é relevante, uma vez que esse tipo de amostra é composta por hidrocarbonetos mais voláteis. Para que massas superiores de petróleo leve pudessem ser introduzidas no sistema de MIC, empregou-se a lã de vidro para retardar a chama e evitar que os gases da combustão fossem liberados rapidamente, aumentando assim a segurança operacional.

Embora os valores de LOQ para MIC tenham sido superiores aos do método de MAE, ambos os métodos foram considerados adequados, uma vez que resultados confiáveis foram obtidos. Os métodos avaliados não requerem a utilização de solventes tóxicos, possuem reduzida manipulação de amostra, são compatíveis com técnicas analíticas, como ICP-OES, ICP-MS e ISE, além de apresentarem uma elevada frequência de análise.

\section{NOMENCLATURA}

IC, cromatografia de íons, do inglês Ion Cromatography

ICP OES, espectrometria de emissão óptica por plasma indutivamente acoplado, do inglês Inductively Coupled Plasma Optical Emission Spectrometry

ICP-MS, espectrometria de massa com plasma indutivamente acoplado, do inglês Inductively Coupled Plasma Mass Spectrometry 
ISE, eletrodo de íon seletivo, do inglês Ion Selective Electrode

LOQ, limite de quantificação, do inglês Limit of Quantification

MAE, extração assistida por micro-ondas, do inglês Microwave-Assisted Extraction

MIC, combustão iniciada por micro-ondas, do inglês Microwave-Induced Combustion

\section{REFERENCIAS}

ANTES, F. G.; DUARTE, F. A.; FLORES, E. L. M.; PANIZ, J. N. G.; FLORES, E. M. M.; DRESSLER, V. L. Preparo de amostras de combustíveis fósseis por piroidrólise para a determinação de flúor e cloro. Química Nova, vol. 33, p. 1130-1134, 2010.

ANTES, F. G.; DUARTE, F. A.; MESKO, M. F.; NUNES, M. A. G.; PEREIRA, V. A.; MULLER, E. I.; DRESSLER, V. L.; FLORES, E. M. M. Determination of toxic elements in coal by ICP-MS after digestion using microwave-induced combustion, Talanta, v. 83, p. 364-369, 2010.

ASTM D 6470-99 (2010), Standard Test Method for Salt in Crude Oils (Potentiometric Method), ASTM International, West Conshohocken, PA, 2010.

BRADLEY, H.B. Petroleum Engineering Handbook, Society of Petroleum Engineers, Texas: Richardson, 1987.

FLORES, E. M. M.; BARIN, J. S.; MESKO, M. F.; KNAPP, G. Sample preparation techniques based on combustion reactions in closed vessels - a brief overview and recent applications, Spectrochimica Acta Part B: Atomic Spectroscopy, v 62, p. 10511064, 2007.

FORTUNY, M.; SILVA, E. B.; FILHO, A. C.; MELO, R. L. F. V.; NELE, M.; COUTINHO, R. C. C.; SANTOS, A. F. Measuring salinity in crude oils: evaluation of methods and an improved procedure. Fuel, v. 87, p. 1241-1248, 2008.

GARY, J. H.; HANDWEK, G. E.; KAISER, M. J. Petroleum Refining - Technology and Economics, 5 ed. Boca Raton: CRC Press Taylor \& Francis Group, 2007.

MACIEL, J. V.; KNORR, C. L., FLORES, E. M. M.; MÜLlER, E. I., MESKO, F. M.; PRIMEL, E. G.; DUARTE, DUAR. A. Feasibility of microwave-induced combustion for trace element determination in Engraulis anchoita by ICP-MS. Food Chemistry, v. 145, p. 927-931, 2014.

MORIGAKI, M. K.; CHIMIN, R. Q. F.; SAD, C. M. S.; FILGUEIRAS, P. R.; CASTRO, E. V. R.; DIAS, J. C. M. Salinity of crude oil: optimization of methodology and new method for extraction of salt in petroleum, Química Nova, v. 33, p. 607-612, 2010.

PEREIRA S. F. J.; MELLO, P. A.; MORAES, D. P.; DUARTE, F. A.; DRESSLER, V. L.; KNAPP, G.; FLORES, E. M. M. Chlorine and sulfur determination in extra-heavy 
crude oil by inductively coupled plasma optical emission spectrometry after microwaveinduced combustion. Spectrochimica Acta B, v. 64, p. 554-558, 2009.

PEREIRA, J. S. F.; MORAES, D. P.; ANTES, F. G.; DIEHL, L. O.; SANTOS, M. F. P.; GUIMARÃES, R. C. L.; FONSECA, T. C. O.; DRESSLER, V. L.; FLORES, E. M. M. Determination of metals and metalloids in light and heavy crude oil by ICP-MS after digestion by microwave-induced combustion. Microchemical Journal, v. 96, p 4-11, 2010 .

PEREIRA, L. S. F. Extração assistida por radiação micro-ondas para a remoção de água, sal e sedimentos de petróleo e posterior caracterização. Dissertação de Mestrado Universidade Federal de Santa Maria, 2012.

SPEIGHT, J. G. Handbook of petroleum product analysis. New Jersey: John Wiley \& Sons, 2002. 